affection in any of its clinical varieties, either attenuated, or even of the bovine type which Musy has tried to put forward.

It is a fact that we have not been fortunate enough up to now to discover the infective agent of Parinaud's disease. But this is not astonishing, so numerous are the diseases where the infective agents are still to be discovered, and we do not see why Parinaud's conjunctivitis and tuberculosis of the conjunctiva should be taken as one, just because they have a few clinical features in common. It would be more scientific, we think, to admit that the clinical features by themselves are not always sufficient to clear up differential diagnosis, and we should rather trust the experimental or histological examination. whenever we meet with a conjunctival affection with involvement of the lymphatic glands. The diagnosis of Parinaud's conjunctivitis should be reserved for those cases where the absence of giant cells, and the negative result of inoculation and culture would justify the elimination of tuberculosis, sporotrichosis, etc., of the conjunctiva.

\title{
TRACHOMA IN THE STRAITS SETTLEMENTS
}

\author{
BY \\ J. KIRK, M.D.Edin., \\ OCULIST TO THE EDINBURGH NATIONAL SERVICE MEDICAL BOARD; \\ FORMERLY VISITING OPHTHALMIC SURGEON, GOVERNMENT HOSPITALS, \\ PENANG, STRAITS SETTLEMENTS.
}

TRACHOMA is the most common and widespread of all the eye affections met with in this part of the world. As the result of twenty years' experience, I should say that the three main causes of blindness among the native population are in the order of their occurrence-trachoma, syphilis, and gonorrhoea, and of the three, trachoma is roughly responsible for 70 per cent. In addition, the amount of eye-maiming and disability short of blindness which it causes is enormous, and not realized. I should like in this paper to bring forward a few personal observations which may be of some interest, though I cannot claim any special originality for them.

Trachoma in the Straits is a disease of the towns, and not so much of the country. It is much more common among artizans, clerks, and the shopkeeping class than in agriculturalists or outdoor workers. There also appears on the surface to be a distinct race susceptibility: the great majority of cases met with are in Chinese. Among the Malay population the ratio of occurrence is very much smaller, and the same may be said of the Tamil or Kling, who come from India in their thousands to this prosperous colony. This great frequency of the disease among the Chinese living in a country 
peopled by several distinct racial types, is certainly a strong argument in favour of racial predisposition. The undoubtedly favourable climatic conditions for the affection are shared equally. Social conditions are not very different, and as regards general physique, the Chinaman is superior to either the Malay or the Kling, yet the underfed, weakly Tamil does not get trachoma, while the sturdy, muscular, well-fed Chinaman does. An interesting fact, perhaps worth mentioning in this connection, is the well-marked tendency to the excess formation of lymphoid tissues in the Mongolian races. A hyperplasia of the follicular tissue of the eyelids may predispose to the development of the trachoma organism. Arguing from the other side against a special racial predisposition, a point of view which personally I am inclined to favour, there is unquestionably no individual or race which does so much to put himself in the way of infection as the Chinaman. $\mathrm{He}$ overcrowds to an appalling extent, and shares all things in common with his fellows in a way which no other race does, and I think all who know him will agree that the factor of a race predisposition to this disease in the Chinese is not really required. If there is any trachoma about he really cannot help getting it. The Malay and the Kling are more particular in their personal habits, especially the former.

As regard the age factor, the disease as met with in children is commonly of the typical granulation or sago-grain type of the textbooks. It is extremely latent in its character, generally' presenting few urgent symptoms, and is often overlooked unless another bacterial infection is superadded. I have seen in small infants a very serious fulminating type of the disease, resulting in corneal ulceration, subsequent opacities, and blindness. This type is probably due to an added gonococcal infection, though I was never able to find the gonococcus in the discharge, which is scanty. My experience has led me to believe that the initial infection of trachoma in the great majority of adult cases dates from early childhood, for I have never to my recollection seen primary infection in an adult Chinese over twenty, and marked changes with secondary infections have generally presented themselves before that date.

In regard to causation, my own opinion is that there is now sufficient reason to believe that the infecting microbe is the cellinclusion body. This is regarded as a protozoal organism, and grouped with the chlamydozoa. It belongs to the class of filtrable viruses, and exists as fine granular bodies, which may be free in the vascular channels, or when taken up by cells cause as a reactionary result a so-called cell inclusion. A most interesting point is the finding of similar bodies in the cells in chronic vaginal and urethral catarrhs, and, to my mind, it seems probable that the original source of trachoma infection may be from such a cause. 
The cell-inclusion bodies in trachoma are very rarely found in the advanced cases. They are much more commonly seen in the early stages of the disease in children.

I wish to point out also, as strongly supporting the cell-inclusion origin of this disease, the essentially chronic course of a case of pure trachoma. The common classification of trachoma into acute and chronic varieties is misleading and inaccurate. Trachoma is a chronic disease, and the acute manifestations so commonly seen are due to a mixed infection. To prove this, I have in the past made a large number of bacteriological examinations of the conjunctival sacs in cases of trachoma. I usually made cultures from the secretion on serum-agar or blood-agar. This is, in my experience, the most satisfactory method of investigating the bacteriology of eye conditions, as it is sometimes very difficult to demonstrate organisms in direct films. The preparations were stained in the usual róutine manner by carbol-thionin and Gram's method. I found that in the advanced complicated cases of trachoma the commonest organism was the Morax bacillus. I found this often in a pure culture, especially where only a sub-acute inflammation existed. In the more acute conditions a mixed infection was more commonstaphylococcus, Weeks' B. and occasionally gonococcus. Some cases presented a combined infection by all four-Morax B., Weeks B., staphylococcus, and gonococcus. The pneumococcus I never found as a secondary infection in trachomatous eyes. The infection by the gonococcus, curiously enough, very rarely gives rise in trachoma to the common acute destructive blennorrhoea. For some reason or other, the result of such an additional infection is generally subacute. The theory that the acute types of trachoma are not pure trachoma, but due to a mixed infection, was thus confirmed. Such a mixed infection is, of course, natural with the ocular tissues already damaged by the chronic devitalizing trachomatous growth and ensuing fibrosis.

The diagnosis of early trachoma is comparatively easy, but in the latter stages naturally must be often difficult. Complications must not be taken for the disease, and an important point to remember is that one is not justified in such cases in diagnosing a Morax or a Weeks infection from the mere presence of these organisms in the conjunctival discharge. The pathognomonic unmistakable signs of trachoma are, first, the typical pannus infiltration and vascularization of the cornea; second, hypertrophy and swelling of the retrotarsal fold of the upper lid; third, the subsequent fibrotic contraction of the conjunctival and adjacent tissues. This pathological tripod is peculiar to trachoma. In no other eye disease is there a similar combination of symptoms. On the other hand, one must avoid the fallacy in trachomatous districts of calling everything trachoma. For instance, pure Weeks's infections are 
found fairly frequently presenting the form of a sub-acute conjunctivitis with a good deal of pus formation. An interesting condition is also that of a gonorrhoeal infection of a chronic nature producing a conjunctival catarrh which is generally longstanding and obstinate to treatment. I have mentioned the acute trachomatous condition in infants due to a mixed infection, but I have also seen cases of an acute infantile blennorrhoea of a non-gonorrhoeal nature with copious discharge leading every now and then to ulceration and corneal opacities. These may be instances of inclusion body blennorrhoea, but I have no microscopical proof to ofier with regard to that.

In conclusion, trachoma, as I have met with it in the adult, is one of the most hopeless of all eye diseases to treat by the usual agents. However, before the pannus and degenerative changes have gone too far, I am fully convinced, that the best line of treatment is surgical, and consists in the complete excision of the retrotarsal fold of the upper lid and of its "cartilage," leaving simply a rim of "cartilage" at the lid margin. This is of course Heisrath's operation. I believe in a large number of cases this operation results in a more or less radical cure: certainly as regards any further progress of the disease to its inevitable goalmore or less complete destruction of the eye and its functions. In the primary stage general local treatment is more successful. This is the time when the "expression" of the nodule helps. Other methods of which I have experience of may be referred to briefly. I am a great believer in the persistent and steady use of the sulphate of copper stick, applying it daily with occasional breaks, but always remembering that where inflammatory and mixed infection conditions are present it acts as an irritant and does harm. This is the stage for other appropriate remedies, zinc sulphate, weak perchloride or bicyanide of mercury, or weak ointments of citrate of copper, and of course suitable sedative applications if there is much pain and iridic inflammation or irritability. Carbonic dioxide snow, Finsen rays, radium, and other cicatrising agents, I have seen give no better results than the much more easily applied and accessible sulphate of copper. In some of the very advanced and hopeless cases of corneal occlusion by opacities and pannus where no other treatment seems to make the least difference, patients often told me that they had received great benefit from sub-conjunctival injections. I generally used 1 to 3,000 of mercury oxycyanate along with acoin. The reaction was generally very marked, but it passed off completely in a few days. I have, at one time or the other, treated at least a hundred patients by this method. I never saw myself that an appreciable difference in the condition ensued, but the sufferers themselves were quite enthusiastic about it, and it is a method of which I should recommend a trial. It is quite 
possible that it does improve in some way, not appreciable objectively, the internal nutrition of the eye structures. Peritomy I used to do often, but it never had any good effect. Optical iridectomies in this condition are practically never successful, and I have tried them frequently. There seems to be so much post-operative lymphexudation following for one thing. Finally, trachoma is a disease which exists only as the result of the absence of the most elementary personal hygiene. If this is present there is no danger of the spread of the infection, and a certainty of its ultimate disappearance from the face of the globe.

\title{
THE MEDICO-LEGAL INTEREST OF A CASE OF BINOCULAR KERATITIS FOLLOWING UNIOCULAR IN JURY*
}

BY

\author{
R. J. Coulter, D.O.Oxon.
}

OPHTHALMIC SURGEON TO THE ROYAL GWENT HOSPITAL, NEWPORT, MON.

CASES of uniocular keratitis following injury have been reported by various observers, but instances in which the second eye has become affected are not so common. In the literature at my disposal I have only been able to find records of such a condition by Ohm, Asmus, and H. Work Dodd (three cases). Owing to the medico-legal interest of the subject I venture to bring the following case to the notice of the Congress :-

H. D. J., marine engineer, aged 25, of good family and personal history, was struck on the outside of the left eye by a piece of steel on November 2, 1915. He reported the accident to the second engineer, but nothing was found in the eye. He was treated by the captain until he reached Savona six days later, where he was treated by an Italian oculist, who said he would try to keep the right eye safe and that it had either come on from disease or from a blow on the eye. He went with his ship to Baltimore, U.S.A.- where he was again under the care of an ophthalmic surgeon-St. John, New Brunswick, Plymouth, Calais, and Cardift, where he was discharged. He came to my out-patient department at the Royal Gwent Hospital on March 6, 1916, when I found the right eye healthy and opacities in the left cornea evidently the result of keratitis. His vision then was right eye $6 / 18-3.5 \mathrm{D}$. cyl. axis $15^{\circ} 6 / 9$; left eye $6 / 36-0.75 \mathrm{D}$. sph. $-5 \mathrm{D}$. cyl. axis $165^{\circ} 6 / 36$. There was a deep-seated central cloud in the cornea with residual vessels in the left eye. The right eye was healthy.

\footnotetext{
* A communication to the Oxford Ophthalmological Congress, July, 1917.
} 